\title{
Time-Dependent Global Attractor for a Class of Nonclassical Parabolic Equations
}

\author{
Fang-hong Zhang \\ Department of Mathematics, Longqiao College of Lanzhou Commercial College, Lanzhou, Gansu 730101, China \\ Correspondence should be addressed to Fang-hong Zhang; zhangfanghong2010@126.com
}

Received 14 May 2014; Revised 12 August 2014; Accepted 13 August 2014; Published 28 August 2014

Academic Editor: Gilles Lubineau

Copyright (c) 2014 Fang-hong Zhang. This is an open access article distributed under the Creative Commons Attribution License, which permits unrestricted use, distribution, and reproduction in any medium, provided the original work is properly cited.

Based on the recent theory of time-dependent global attractors in the works of Conti et al. (2013) and di Plinio et al. (2011), we prove the existence of time-dependent global attractors as well as the regularity of the time-dependent global attractor for a class of nonclassical parabolic equations.

\section{Introduction}

Let $\Omega$ be a bounded set of $\mathbb{R}^{3}$ with smooth boundary $\partial \Omega$. For any $\tau \in \mathbb{R}$, we consider the following nonclassical equation:

$$
\begin{gathered}
\varepsilon(t) u_{t}-\Delta u_{t}-\Delta u+f(u)=g(x), \quad \text { in } \Omega \times[\tau, \infty), \\
u=0, \quad \text { on } \partial \Omega, \\
u(x, \tau)=a(x), \quad x \in \Omega,
\end{gathered}
$$

where $a: \Omega \rightarrow \mathbb{R}$ are assigned data and $\varepsilon(t)$ is a decreasing bounded function satisfying

$$
\lim _{t \rightarrow+\infty} \varepsilon(t)=0,
$$

and $L>0$ is such that

$$
\sup _{t \in \mathbb{R}}|\varepsilon(t)| \leq L .
$$

The nonlinearity $f \in \mathscr{C}^{2}(\mathbb{R})$, with $f(0)=0$, is assumed to satisfy the inequality

$$
\left|f^{\prime \prime}(s)\right| \leq c(1+|u|), \quad \text { for some } c \geq 0,
$$

along with the dissipation condition

$$
\liminf _{|s| \rightarrow \infty} \frac{f(s)}{s}>-\lambda_{1},
$$

where $\lambda_{1}$ is the first eigenvalue of $-\Delta$ in $H_{0}^{1}(\Omega)$ and $g(x) \epsilon$ $L^{2}(\Omega)$.

The classical reaction diffusion equation has a long history in mathematical physics and appears in many mathematical models. It arises in several bead mark problems of hydrodynamics and heat transfer theory, such as heat transfer, as well as in solid-fluid in Hradion configurations and, of course, in standard situations mass diffusion and flow through porous media [1, 2]. In 1980, Aifantis in [1] pointed out that the classical diffusion equation does not suffice to describe transport in media with two temperatures or two diffusions as well as in cases where the diffusions substances behave as a viscous fluid. It turns out that new terms appear in the classical diffusion equation when such effects are considered. In particular, the mixed spatiotemporal derivative $\Delta u_{t}$ consistently appears in several generalized reaction-diffusion models $[1,3-6]$ and this is our motivation for studying (1) which contains, in addition, the nonlinear term $f(u)$ and inhomogeneous term $g(x)$, along with the time-dependent parameter $\varepsilon(t)$. The presence of the $\Delta u_{t}$ has some important consequences on the character of the solution of the partial differential equation under consideration. For example, the classical reaction diffusion equation has smoothing effect; for example, although the initial data only belongs to a weaker topology space, the solution will belong to a stronger topology space with higher regularity. However, for (1), if the initial data $u_{0}$ belongs to $H_{0}^{1}(\Omega)$, then the solution $u(t, x)$ with 
$u(0, x)=u_{0}$ is always in $H_{0}^{1}(\Omega)$ and has no higher regularity because of the term $-\Delta u_{t}$.

In the case when $\varepsilon(t)=\varepsilon$ is a positive constant, the asymptotic behavior of solutions to (1) has been extensively studied by several authors in $[2,7-16]$ and the references therein. In the general case of time dependence, that is, $\varepsilon=\varepsilon(t)$, the longtime behavior of the nonclassical equation has not been considered so far. In this paper, we borrow some ideas from the following previous contributors: Conti et al. in [17] who introduced the theory of time-dependent global attractors and apply the theory to the wave equations; Caraballo et al. who introduced a one-parameter family of Banach spaces in the context of cocycles for nonautonomous and random dynamical systems in [18] as well as time-dependent spaces [19] in the context of stochastic partial differential equations; and Flandoli and Schmalfuss in [20] who introduced a family of metric spaces depending on a parameter and applied it to the stochastic form of Navier-Stokes equations. In this paper, based on the recent theory of time-dependent global attractors of Conti et al. [17] and di Plinio et al. [21], we prove the existence of time-dependent global attractors as well as the regularity of the time-dependent global attractor for a class of nonclassical parabolic equations as described by (1).

The paper is organized as follows. In Section 2, we present some preliminaries, establish the necessary notation and functions spaces to be used in the subsequent analysis, and give some useful lemmas. In Section 3, we prove the existence of time-dependent global attractors for the nonclassical parabolic equation and its regularity. Our main results are Theorems 14 and 16.

\section{Preliminaries}

In this section, we introduce some notations and definitions, along with a lemma.

We set $H=L^{2}(\Omega)$, with inner product $\langle\cdot, \cdot\rangle$ and norm $\|\cdot\|$. For $0 \leq \sigma \leq 2$, we define the hierarchy of (compactly) nested Hilbert spaces:

$$
\begin{gathered}
H_{\sigma}=\operatorname{dom}\left(A^{\sigma / 2}\right), \\
\langle w, v\rangle_{\sigma}=\left\langle A^{\sigma / 2} w, A^{\sigma / 2} v\right\rangle, \\
\|w\|_{\sigma}=\left\|A^{\sigma / 2} w\right\| .
\end{gathered}
$$

Then, for $t \in \mathbb{R}$ and $0 \leq \sigma \leq 2$, we introduce the timedependent spaces $\mathscr{H}_{t}^{\sigma}=H_{\sigma+1}$ endowed with the timedependent product norms:

$$
\|u\|_{\mathscr{H}_{t}^{\sigma}}^{2}=\varepsilon(t)\|u\|_{\sigma}^{2}+\|u\|_{\sigma+1}^{2}
$$

The symbol $\sigma$ is always omitted whenever zero. In particular, the time-dependent phase space where we settle the problem is

$$
\mathscr{H}_{t}=H_{1} \quad \text { with }\|u\|_{\mathscr{H}_{t}}^{2}=\varepsilon(t)\|u\|^{2}+\|u\|_{1}^{2} .
$$

Then, we have the compact embeddings:

$$
\mathscr{H}_{t}^{\sigma} \subset \mathscr{H}_{t}, \quad 0<\sigma \leq 2,
$$

with injection constants independent of $t \in \mathbb{R}$.

Note that the spaces $\mathscr{H}_{t}$ are all the same as linear spaces, and the norms $\|z\|_{\mathscr{H}_{t}}^{2}$ and $\|\cdot\|_{\mathscr{H}_{\tau}}^{2}$ are equivalent for any fixed $t, \tau \in \mathbb{R}$.

According to (5), we have the following lemma.

Lemma 1. The following inequalities hold for some $0<v<1$ and $c_{1} \geq 0$ :

$$
\begin{gathered}
2\langle F(u), 1\rangle \geq-(1-v)\|u\|_{1}^{2}-c_{1}, \\
\langle f(u), u\rangle \geq-(1-v)\|u\|_{1}^{2}-c_{1}, \quad \forall u \in H_{1} .
\end{gathered}
$$

\section{Existence of the Time-Dependent Global Attractor}

3.1. Well-Posedness. For any $\tau \in \mathbb{R}$, we rewrite the problem (1) as

$$
\begin{gathered}
\varepsilon(t) u_{t}+A u_{t}+A u+f(u)=g(x), \quad t>\tau, \\
u(x, \tau)=a .
\end{gathered}
$$

Using the Galerkin approximation method, we can obtain the following result concerning the existence and uniqueness of solutions; see, for example, [2, 7, 8, 15, 16].

Lemma 2. Under the assumptions of (2)-(5), for any a $\in \mathscr{H}_{\tau}$, there is a unique solution $u$ of $(1)$, on any interval $[\tau, t]$ with $t \geq \tau$,

$$
u \in \mathscr{C}\left([\tau, t] ; H_{1}\right)
$$

Furthermore, for $i=1,2$, let $u_{\tau}^{i} \in \mathscr{H}_{\tau}$ be two initial conditions such that $\left\|u_{\tau}^{i}\right\|_{\mathscr{H}_{\tau}} \leq R$ and denote by $u_{i}$ the corresponding solutions to the problem of (11). Then the following estimates hold as follows:

$$
\left\|u_{1}(t)-u_{2}(t)\right\|_{\mathscr{H}_{t}}^{2} \leq e^{K(t-\tau)}\left\|u_{\tau}^{1}-u_{\tau}^{2}\right\|_{\mathscr{H}_{t}}^{2}, \quad t \geq \tau
$$

for some constant $K=K(R) \geq 0$.

According to Lemma 2 above, the family of maps with $t \geq$ $\tau \in \mathbb{R}$

$$
U(t, \tau): \mathscr{H}_{\tau} \longrightarrow \mathscr{H}_{t}
$$

acting as

$$
u_{\tau} \longrightarrow u(t)=U(t, \tau) z_{\tau}
$$

where $u$ is the unique solution of (11) with initial time $\tau$ and initial condition $u_{\tau}=\mathscr{H}_{\tau}$, defines a strongly continuous process on the family $\left\{\mathscr{H}_{t}\right\}_{t \in \mathbb{R}}$. 


\subsection{Time-Dependent Absorbing Set}

Definition 3. A time-dependent absorbing set for the process $U(t, \tau)$ is a uniformly bounded family $\mathscr{B}=\left\{B_{t}\right\}_{t \in \mathbb{R}}$ with the following property: for every $R \geq 0$ there exists $\theta_{e}=\theta_{e}(R) \geq 0$ such that

$$
\tau \leq t-\theta_{e} \Longrightarrow U(t, \tau) \mathbb{B}_{\tau}(R) \subset B_{t} .
$$

Lemma 4. Under the assumptions of (2)-(5), for $u_{\tau} \in \mathscr{H}_{\tau}, t \geq$ $\tau$, let $U(t, \tau) u_{\tau}$ be the solution of (1); then, there exist positive constants $\delta, C_{\nu,\|g\|}$ and an increasing positive function $\mathbb{Q}$ such that

$$
\left\|U(t, \tau) u_{\tau}\right\|_{\mathscr{H}_{t}} \leq \mathbb{Q}\left(\left\|u_{\tau}\right\|_{\mathscr{H}_{\tau}}\right) e^{-\delta(t-\tau)}+C_{\delta, v,\|g\|}, \quad \forall t \geq \tau .
$$

Proof. Multiplying (11) by $u$, we obtain

$$
\begin{gathered}
\frac{\mathrm{d}}{\mathrm{d} t}\left(\varepsilon\|u\|^{2}+2\|\nabla u\|^{2}\right)+2\|\nabla u\|^{2}-\varepsilon^{\prime}\|u\|^{2}+2\langle f(u), u\rangle \\
-2\langle u, g\rangle=0 .
\end{gathered}
$$

Noting that $\varepsilon^{\prime}<0$ and using (3) and Young and Poincaré inequalities, for $\delta>0$ small, we infer that

$$
\frac{\mathrm{d}}{\mathrm{d} t}\left(\varepsilon\|u\|^{2}+2\|\nabla u\|^{2}\right)+\delta\left(\varepsilon\|u\|^{2}+\|\nabla u\|^{2}\right) \leq C_{\delta, v,\|g\|} .
$$

By the Gronwall lemma, we have

$$
\|u(t)\|_{\mathscr{H}_{t}}^{2} \leq C e^{-\delta(t-\tau)}\|u(\tau)\|_{\mathscr{H}_{t}}^{2}+C_{\delta, v,\|g\|} \cdot
$$

This completes the proof.

Lemma 5 (time-dependent absorbing set). Under the assumptions of (2)-(5), there exists a constant $R_{1}>0$, such that the family $\mathfrak{B}=\left\{\mathbb{B}_{t}\left(R_{1}\right)\right\}_{t \in \mathbb{R}}$ is a time-dependent absorbing set for $U(t, \tau)$.

Proof. From the proof of Lemma 4 , for $u \in \mathbb{B}_{\tau}(R)$, there exists $\theta_{e} \geq 0$, provided that $t-\tau \geq \theta_{e}$,

$$
\left\|U(t, \tau) u_{\tau}\right\|_{\mathscr{H}_{t}} \leq R_{0} .
$$

This concludes the proof of the existence of the timedependent absorbing set.

We can assume that the time-dependent absorbing set $\mathfrak{B}_{t}=\mathbb{B}_{t}\left(R_{1}\right)$ is positively invariant (namely, $U(t, \tau) B_{\tau} \subset B_{t}$ for all $t \geq \tau)$. Indeed, calling $\theta_{e}$ the entering time of $B_{t}$ such that

$$
U(t, \tau) B_{\tau} \subset B_{t}, \quad \forall \tau \leq t-\theta_{e},
$$

we can substitute $B_{t}$ with the invariant absorbing family:

$$
\bigcup_{\tau \leq t-\theta_{e}} U(t, \tau) B_{\tau} \subset B_{t} .
$$

3.3. Time-Dependent Global Attractor. As introduced in [17], for $t \in \mathbb{R}$, let $X_{t}$ be a family of normed spaces; we consider the collection

$$
\begin{gathered}
\mathbb{K}=\left\{\mathfrak{R}=\left\{K_{t}\right\}_{t \in \mathbb{R}}: K_{t} \subset X_{t}\right. \text { compact, } \\
\Re \text { pullback attracting }\} .
\end{gathered}
$$

When $\mathbb{K} \neq \varnothing$ we say that the process is asymptotically compact.

Definition 6. One calls a time-dependent global attractor the smallest element of $\mathbb{K}$; that is, the family $\mathfrak{A}=\left\{A_{t}\right\}_{t \in \mathbb{R}} \in \mathbb{K}$ such that

$$
A_{t} \subset K_{t}, \quad \forall t \in \mathbb{R}
$$

for any element $\Re=\left\{K_{t}\right\}_{t \in \mathbb{R}} \in \mathbb{K}$.

Theorem 7 (see [17]). If $U(t, \tau)$ is asymptotically compact, then the time-dependent attractor $\mathfrak{A}$ exists and coincides with the set $\mathfrak{A}=\left\{A_{t}\right\}_{t \in \mathbb{R}}$. In particular, it is unique.

According to Definition 6, the existence of the timedependent global attractor will be proved by a direct application of the abstract Theorem 7. Precisely, in order to show that the process is asymptotically compact, we will exhibit a pullback attracting family of compact sets. To this aim, the strategy classically consists in finding a suitable decomposition of the process in the sum of a decaying part and of a compact one.

3.3.1. The First Decomposition of the System Equations. For the nonlinearity $f$, following $[11,15,17]$, we decompose $f$ as follows:

$$
f=f_{0}+f_{1}
$$

where $f_{0}, f_{1} \in \mathscr{C}^{2}(\mathbb{R})$ satisfy, for some $c \geq 0$,

$$
\begin{gathered}
\left|f_{0}^{\prime \prime}(u)\right| \leq c(1+|u|), \quad \forall u \in \mathbb{R}, \\
f_{0}(u) u \geq 0, \quad \forall u \in \mathbb{R}, \\
f_{0}(0)=f_{0}^{\prime}(0)=0, \\
\left|f_{1}^{\prime}(u)\right| \leq c, \quad \forall u \in \mathbb{R} .
\end{gathered}
$$

Noting that $\mathfrak{B}=\left\{\mathbb{B}_{t}\left(R_{1}\right)\right\}_{t \in \mathbb{R}}$ is a time-dependent absorbing set for $U(t, \tau) u_{\tau}$, then for each initial data $u_{\tau} \in$ $\mathbb{B}_{\tau}\left(R_{1}\right)$, we decompose $U(t, \tau)$ as

$$
U(t, \tau) u_{\tau}=U_{1}(t, \tau) u_{\tau}+U_{2}(t, \tau) u_{\tau},
$$

where $v=U_{1}(t, \tau) u_{\tau}$ and $w=U_{2}(t, \tau) u_{\tau}$ solve the following equations, respectively:

$$
\begin{gathered}
\varepsilon(t) v_{t}+A v_{t}+A v+f_{0}(v)=0, \\
U_{1}(\tau, \tau)=u_{\tau}, \\
\varepsilon(t) w_{t}+A w_{t}+A w+f(u)-f_{0}(v)=g, \\
U_{2}(\tau, \tau)=0 .
\end{gathered}
$$


Lemma 8. Under assumptions of (2)-(5), (27)-(30), there exists $\delta=\delta(\mathfrak{B})>0$ such that

$$
\left\|U_{1}(t, \tau) u_{\tau}\right\|_{\mathscr{H}_{t}} \leq C_{3} e^{-\delta(t-\tau)}, \quad \forall t \geq \tau .
$$

Proof. Multiplying (32) by $v$, we obtain

$$
\frac{\mathrm{d}}{\mathrm{d} t}\left(\varepsilon\|v\|^{2}+\|\nabla v\|^{2}\right)-\varepsilon^{\prime}\|v\|^{2}+2\|\nabla v\|^{2}+2\langle f(v), v\rangle=0 .
$$

Using (28) and noting that $\varepsilon^{\prime}<0$, by Young and Poincaré inequalities, for $\delta>0$ small, we infer

$$
\frac{\mathrm{d}}{\mathrm{d} t}\left(\varepsilon\|v\|^{2}+\|\nabla v\|^{2}\right)+\delta\left(\varepsilon\|v\|^{2}+\|\nabla v\|^{2}\right) \leq 0 .
$$

By the Gronwall lemma, we complete the proof.

Remark 9. From Lemmas 4 and 8, we have the uniform bound

$$
\sup _{t \geq \tau}\left[\|U(t, \tau)\|_{\mathscr{H}_{t}}+\left\|U_{1}(t, \tau)\right\|_{\mathscr{H}_{t}}+\left\|U_{2}(t, \tau)\right\|_{\mathscr{H}_{t}}\right] \leq C .
$$

Lemma 10. Under the assumptions of (2)-(5), (27)-(30), there exists $M=M(\mathfrak{B})>0$ such that

$$
\sup _{t \geq \tau}\left\|U_{2}(t, \tau) u_{\tau}\right\|_{\mathscr{H}_{t}^{1 / 3}} \leq M
$$

Proof. Multiplying (33) by $A^{(1 / 3)} w$, we obtain

$$
\begin{aligned}
\frac{\mathrm{d}}{\mathrm{d} t}(\varepsilon & \left.\left\|A^{1 / 6} w\right\|^{2}+\left\|A^{2 / 3} w\right\|^{2}\right)+2\left\|A^{2 / 3} w\right\|^{2} \\
& +2\left\langle f(u)-f_{0}(v)-g, A^{1 / 3} w\right\rangle=\varepsilon^{\prime}\left\|A^{1 / 6} w\right\|^{2} .
\end{aligned}
$$

In view of Remark 9 and the growth of $f$ and $f_{0}$, using the embedding $H_{1} \subset L^{6}(\Omega)$, we have

$$
\begin{aligned}
2\left|\left\langle f(u), A^{1 / 3} w\right\rangle\right| & \leq c \int_{\Omega}\left(1+|u|^{3}\right)\left|A^{1 / 3} w\right| \\
& \leq c\left(1+\|u\|_{L^{6}}^{3}\right)\left\|A^{1 / 3} w\right\| \\
& \leq c\left\|A^{1 / 3} w\right\| \\
& \leq \frac{1}{4}\left\|A^{2 / 3} w\right\|^{2}+C \\
2\left|\left\langle f_{0}(v)+g, A^{1 / 3} w\right\rangle\right| & \leq c\left\|A^{1 / 3} w\right\| \leq \frac{1}{4}\left\|A^{2 / 3} w\right\|^{2}+C_{\|g\|} \cdot
\end{aligned}
$$

Noting that $\varepsilon^{\prime}<0$, by Young and Poincaré inequalities, for $\delta>0$ small, we infer

$$
\begin{aligned}
& \frac{\mathrm{d}}{\mathrm{d} t}\left(\varepsilon\left\|A^{1 / 6} w\right\|^{2}+\left\|A^{2 / 3} w\right\|^{2}\right)+\delta\left(\varepsilon\left\|A^{1 / 6} w\right\|^{2}+\left\|A^{2 / 3} w\right\|^{2}\right) \\
& \quad \leq C .
\end{aligned}
$$

By the Gronwall lemma, we complete the proof.
Remark 11. From Lemma 10, we immediately have the following regularity result: $\mathscr{A}_{t}$ is bounded in $\mathscr{H}_{t}^{1 / 3}$ (with a bound independent of $t$ ).

Theorem 12 (see [17]). If $U(t, \tau)$ is a $T$-closed process for some $T>0$, which possesses a time-dependent global attractor $\mathfrak{A}$, then $\mathfrak{A}$ is invariant.

Remark 13 (see [17]). If the process $U(t, \tau)$ is closed, it is $T$ closed, for any $T>0$. Note that if the process $U(t, \tau)$ is a continuous (or even norm-to-weak continuous) map for all $t \geq \tau$, then the process is closed.

Theorem 14 (existence of the time-dependent global attractor). Under the assumptions of (2)-(5), the process $U(t, \tau)$ : $\mathscr{H}_{\tau} \rightarrow \mathscr{H}_{t}$ generated by problem (1) admits an invariant timedependent global attractor $\mathfrak{A}=\left\{A_{t}\right\}_{t \in \mathbb{R}}$.

According to Lemma 10, we consider the family $\Re=$ $\left\{K_{t}\right\}_{t \in \mathbb{R}}$, where

$$
K_{t}=\left\{u \in \mathscr{H}_{t}^{1 / 3}:\|u\|_{\mathscr{H}_{t}^{1 / 3}} \leq M\right\},
$$

where $K_{t}$ is compact by the compact embedding $\mathscr{H}_{t}^{1 / 3} \subset \mathscr{H}_{t}$; besides, since the injection constants are independent of $t$, $\Re$ is uniformly bounded. Hence, according to Lemmas 5,8 , and $10, \Re$ is pullback attracting, and the process $U(t, \tau)$ is asymptotically compact, which proves the existence of the unique time-dependent global attractor. In order to state the invariance of the time-dependent global attractor, due to the strong continuity of the process $U(t, \tau)$ stated in Lemma 2, according to Remark 13, the process $U(t, \tau)$ is closed, and it is $T$-closed, for some $T>0$; then by Theorem 12 , we know that the time-dependent global attractor $\mathfrak{A}$ is invariant.

\subsection{Regularity of the Time-Dependent Global Attractor}

3.4.1. The Second Decomposition of the System Equations. We fix $\tau \in \mathbb{R}$ and each initial data $u_{\tau} \in \mathbb{A}_{\tau}$, decomposing $U(t, \tau)$ as

$$
U(t, \tau) u_{\tau}=U_{3}(t, \tau) u_{\tau}+U_{4}(t, \tau) u_{\tau},
$$

where $v=U_{3}(t, \tau) u_{\tau}$ and $w=U_{4}(t, \tau) u_{\tau}$ solve the following equations, respectively:

$$
\begin{gathered}
\varepsilon(t) v_{t}+A v_{t}+A v=0, \\
U_{3}(\tau, \tau)=u_{\tau}, \\
\varepsilon(t) w_{t}+A w_{t}+A w+f(u)=g, \\
U_{4}(\tau, \tau)=0 .
\end{gathered}
$$

As a particular case of Lemma 8, we learn that

$$
\left\|U_{3}(t, \tau) u_{\tau}\right\|_{\mathscr{H}_{t}} \leq C_{4} e^{-\delta(t-\tau)}, \quad \forall t \geq \tau .
$$

Lemma 15. Under assumptions of (2)-(5), for some $M=$ $M(\mathfrak{A})>0$, one has the uniform bound

$$
\sup _{t \geq \tau}\left\|U_{4}(t, \tau) u_{\tau}\right\|_{\mathscr{H}_{t}^{1}} \leq M \text {. }
$$


Proof. Multiplying (46) by $A w_{t}+A w$, we obtain

$$
\begin{gathered}
\frac{\mathrm{d}}{\mathrm{d} t}\left(\varepsilon\left\|A^{1 / 2} w\right\|^{2}+2\|A w\|^{2}-2\langle g, A w\rangle\right)+2 \varepsilon\left\|A^{1 / 2} w_{t}\right\|^{2} \\
+2\left\|A w_{t}\right\|^{2}+2\|A w\|^{2}+2\langle f(u), A w\rangle \\
-2\langle g, A w\rangle+2\left\langle f(u), A w_{t}\right\rangle=\varepsilon^{\prime}\left\|A^{1 / 2} w\right\|^{2} .
\end{gathered}
$$

We denote

$$
\mathscr{M}(t)=\varepsilon\left\|A^{1 / 2} w\right\|^{2}+2\|A w\|^{2}-2\langle g, A w\rangle,
$$

noting that

$$
c\|w\|_{\mathscr{H}_{t}^{1}}^{2}-C_{6} \leq \mathscr{M}(t) \leq c\|w\|_{\mathscr{H}_{t}^{1}}^{2}+C_{7} .
$$

Noting that (2) and using Young and Poincaré inequalities, for $\delta>0$ small, we infer

$$
\begin{aligned}
\frac{\mathrm{d}}{\mathrm{d} t} \mathscr{M}(t) & +\delta \mathscr{M}(t)+2 \varepsilon\left\|A^{1 / 2} w_{t}\right\|^{2}+2\left\|A w_{t}\right\|^{2} \\
\leq & -2\langle f(u), A w\rangle-(2+2 \delta)\langle g, A w\rangle \\
& -2\left\langle f(u), A w_{t}\right\rangle+\varepsilon^{\prime}\left\|A^{1 / 2} w\right\|^{2} .
\end{aligned}
$$

Denoting by $C>0$ a generic constant depending on the size of $A_{t}$ in $\mathscr{H}_{t}^{1 / 3}$, using the invariance of the attractor, we find

$$
\|U(t, \tau) u\|_{\mathscr{H}_{t}^{1 / 3}} \leq C .
$$

Exploiting the embeddings $H_{1 / 3} \subset L^{18 / 7}, H_{4 / 3} \subset L^{18}$, we get

$$
\|f(u)\|_{1} \leq\left\|f^{\prime}(u)\right\|_{L^{9}}\left\|A^{1 / 2} u\right\|_{L^{18 / 7}} \leq C\left(1+\|u\|_{L^{18}}\right) \leq C ;
$$

this yields

$$
\begin{gathered}
-2\langle f(u), A w\rangle-(2+2 \delta)\langle g, A w\rangle \\
\leq C_{\delta,\|g\|}\|A w\| \leq 2\|A w\|^{2}+C, \\
-2\left\langle f(u), A w_{t}\right\rangle \leq C\left\|A w_{t}\right\| \leq 2\left\|A w_{t}\right\|^{2}+C ;
\end{gathered}
$$

noting that $\varepsilon^{\prime}<0$, we infer

$$
\frac{\mathrm{d}}{\mathrm{d} t} \mathscr{M}(t)+\delta \mathscr{M}(t) \leq C .
$$

By the Gronwall lemma, we can get (48) immediately.

This completes the proof.

Therefore, we have the following regularity result.

Theorem 16 (regularity of the time-dependent global attractor). Under the assumptions of (2)-(5), the time-dependent global attractor $\mathfrak{A}=\left\{A_{t}\right\}_{t \in \mathbb{R}}, A_{t}$ is bounded in $\mathscr{H}_{t}^{1}$, with a bound independent of $t$.
In fact, we define

$$
E_{t}=\left\{u \in \mathscr{H}_{t}^{1}:\|u\|_{\mathscr{H}_{t}^{1}} \leq M_{1}\right\} ;
$$

according to inequalities (47) and (48), for all $t \in \mathbb{R}$, we have

$$
\lim _{\tau \rightarrow-\infty} \operatorname{dist}\left(U(t, \tau) A_{\tau}, E_{t}\right)=0,
$$

where dist denotes the Hausdorff semidistance in $\mathscr{H}_{t}$; that is,

$$
\operatorname{dist}(B, C)=\sup _{x \in B} \operatorname{dist}_{\mathscr{\ell}_{t}}(x, C)=\sup _{x \in B} \inf _{y \in C}\|x-y\|_{\mathscr{H}_{t}} .
$$

From Theorem 14, we know that the time-dependent global attractor $\mathfrak{A}=\left\{A_{t}\right\}_{t \in \mathbb{R}}$ is invariant; this means that

$$
\operatorname{dist}\left(A_{t}, E_{t}\right)=0 \text {. }
$$

Hence, $A_{t} \subset \overline{E_{t}}=E_{t}$; that is, $A_{t}$ is bounded in $\mathscr{H}_{t}^{1}$, with a bound independent of $t$.

\section{Conflict of Interests}

The author declares that there is no conflict of interests regarding the publication of this paper.

\section{Acknowledgments}

The author expresses her sincere thanks to the anonymous reviewer for his/her careful reading of the paper, giving valuable comments and suggestions. She also thanks the editors for their kind help.

\section{References}

[1] E. C. Aifantis, "On the problem of diffusion in solids," Acta Mechanica, vol. 37, no. 3-4, pp. 265-296, 1980.

[2] R. Temam, Infinite-Dimensional Dynamical Systems in Mechan$i$ ics and Physic, Springer, New York, NY, USA, 1997.

[3] J. L. Lions and E. Magenes, Non-Homogeneous Boundary Value Problems and Applications, Spring, Berlin, Germany, 1972.

[4] K. Kuttler and E. C. Aifantis, "Existence and uniqueness in nonclassical diffusion," Quarterly of Applied Mathematics, vol. 45, no. 3, pp. 549-560, 1987.

[5] K. Kuttler and E. Aifantis, "Quasilinear evolution equations in nonclassical diffusion," SIAM Journal on Mathematical Analysis, vol. 19, no. 1, pp. 110-120, 1988.

[6] E. C. Aifantis, "Gradient nanomechanics: applications to deformation, fracture, and diffusion in nanopolycrystals," Metallurgical and Materials Transactions A, vol. 42, no. 10, pp. 2985-2998, 2011.

[7] V. K. Kalantarov, "On the attractors for some non-linear problems of mathematical physics," Zapiski Nauchnykh Seminarov Leningradskogo Otdeleniya Matematicheskogo Instituta imeni $V$. A. Steklova Akademii Nauk SSSR (LOMI), vol. 152, pp. 50-54, 1986.

[8] Y. Xiao, "Attractors for a nonclassical diffusion equation," Acta Mathematicae Applicatae Sinica, vol. 18, no. 2, pp. 273-276, 2002.

[9] C. Y. Sun, S. Y. Wang, and C. K. Zhong, "Global attractors for a nonclassical diffusion equation," Acta Mathematica Sinica, English Series, vol. 23, no. 7, pp. 1271-1280, 2007. 
[10] S. Y. Wang, D. S. Li, and C. K. Zhong, "On the dynamics of a class of nonclassical parabolic equations," Journal of Mathematical Analysis and Applications, vol. 317, no. 2, pp. 565-582, 2006.

[11] C. Sun and M. Yang, "Dynamics of the nonclassical diffusion equations," Asymptotic Analysis, vol. 59, no. 1-2, pp. 51-81, 2008.

[12] Y. F. Liu and Q. Z. Ma, "Exponential attractors for a nonclassical diffusion equation," Electronic Journal of Differential Equations, vol. 9, pp. 1-9, 2009.

[13] Q. Z. Ma, Y. F. Liu, and F. H. Zhang, "Global attractors in $H^{1}\left(\mathbb{R}^{N}\right)$ for nonclassical diffusion equations," Discrete Dynamics in Nature and Society, vol. 2012, Article ID 672762, 16 pages, 2012.

[14] H. Q. Wu and Z. Y. Zhang, "Asymptotic regularity for the nonclassical diffusion equation with lower regular forcing term," Dynamical Systems, vol. 26, no. 4, pp. 391-400, 2011.

[15] L.-X. Pan and Y.-F. Liu, "Robust exponential attractors for the non-autonomous nonclassical diffusion equation with memory," Dynamical Systems, vol. 28, no. 4, pp. 501-517, 2013.

[16] F.-H. Zhang and Y.-F. Liu, "Pullback attractors in $H^{1}\left(\mathbb{R}^{N}\right)$ for non-autonomous nonclassical diffusion equations," Dynamical Systems, vol. 29, no. 1, pp. 106-118, 2014.

[17] M. Conti, V. Pata, and R. Temam, "Attractors for processes on time-dependent spaces. Applications to wave equations," Journal of Differential Equations, vol. 255, no. 6, pp. 1254-1277, 2013.

[18] T. Caraballo, M. J. Garrido-Atienza, and B. Schmalfuss, "Existence of exponentially attracting stationary solutions for delay evolution equations," Discrete and Continuous Dynamical Systems B, vol. 18, no. 2-3, pp. 271-293, 2007.

[19] T. Caraballo, P. E. Kloeden, and B. Schmalfuß, "Exponentially stable stationary solutions for stochastic evolution equations and their perturbation," Applied Mathematics and Optimization, vol. 50, no. 3, pp. 183-207, 2004.

[20] F. Flandoli and B. Schmalfuss, "Random attractors for the 3D stochastic Navier-Stokes equation with multiplicative white noise," Stochastics and Stochastics Reports, vol. 59, no. 1-2, pp. 21-45, 1996.

[21] F. di Plinio, G. S. Duane, and R. Temam, "Time-dependent attractor for the oscillon equation," Discrete and Continuous Dynamical Systems A, vol. 29, no. 1, pp. 141-167, 2011. 


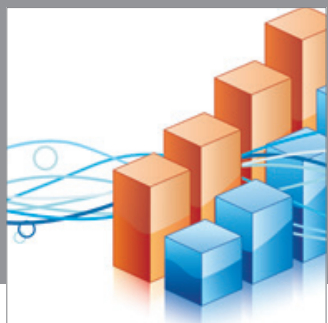

Advances in

Operations Research

mansans

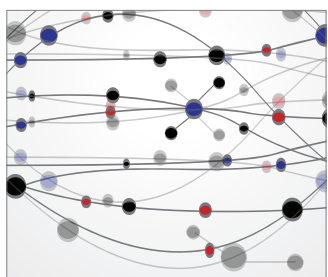

The Scientific World Journal
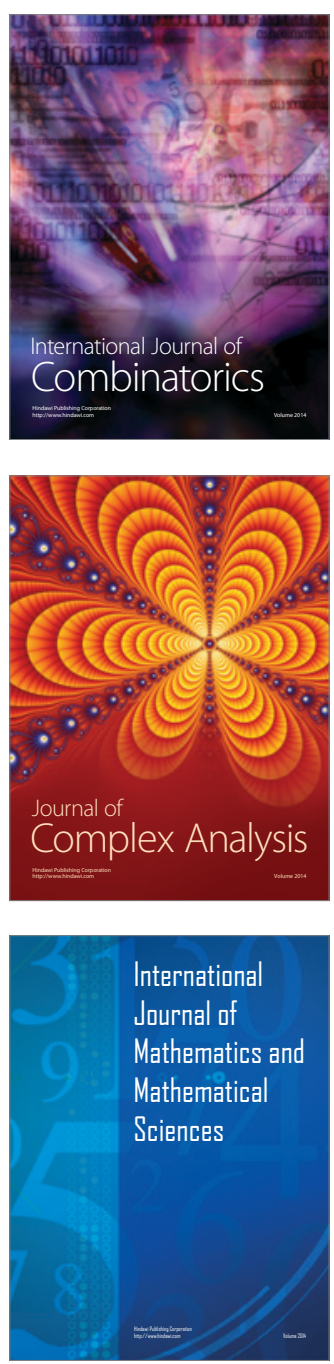
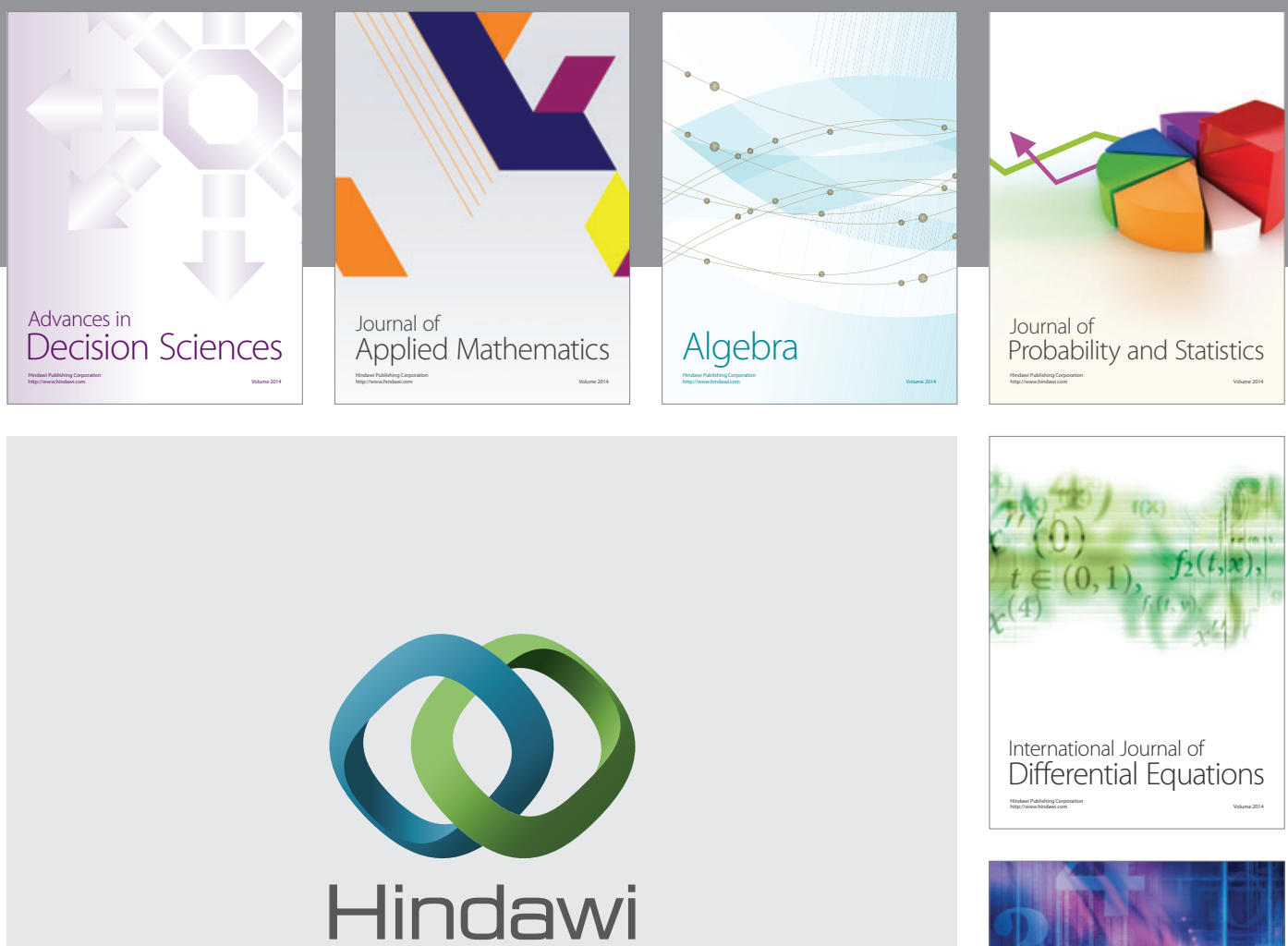

Submit your manuscripts at http://www.hindawi.com
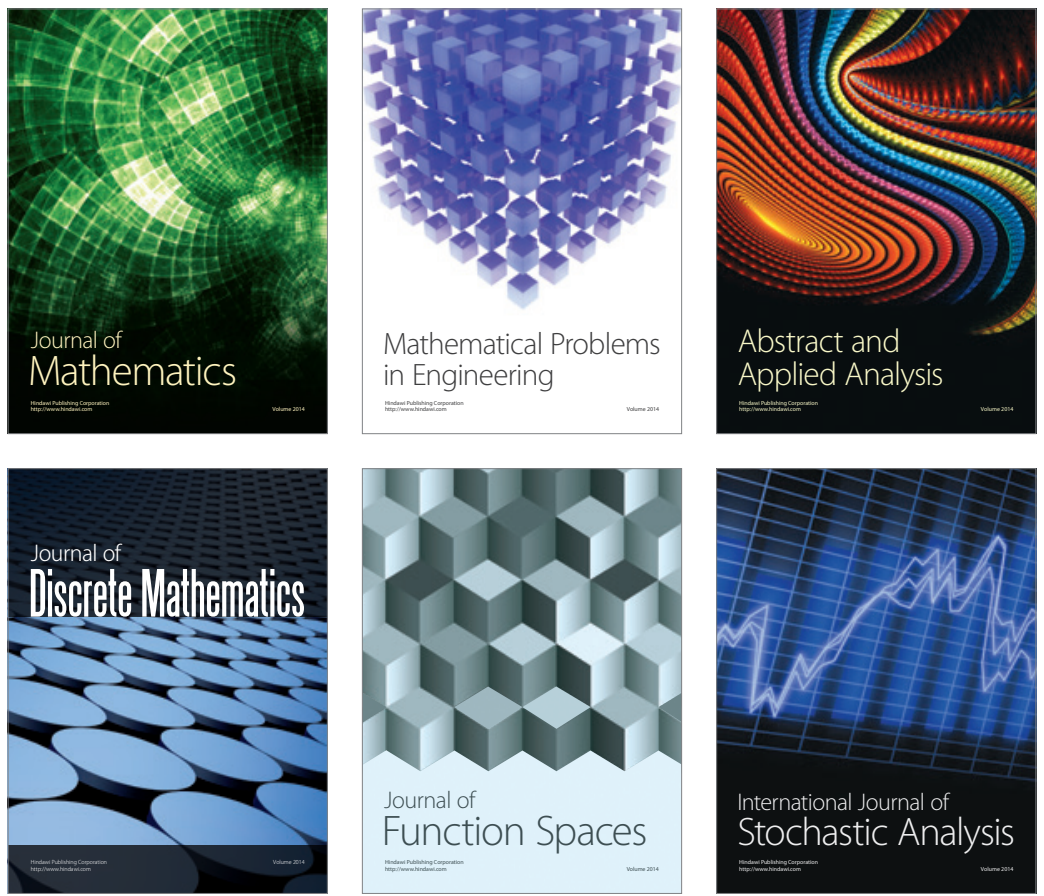

Journal of

Function Spaces

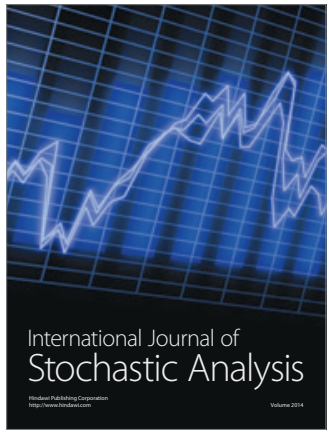

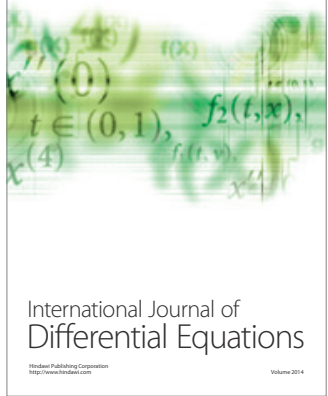
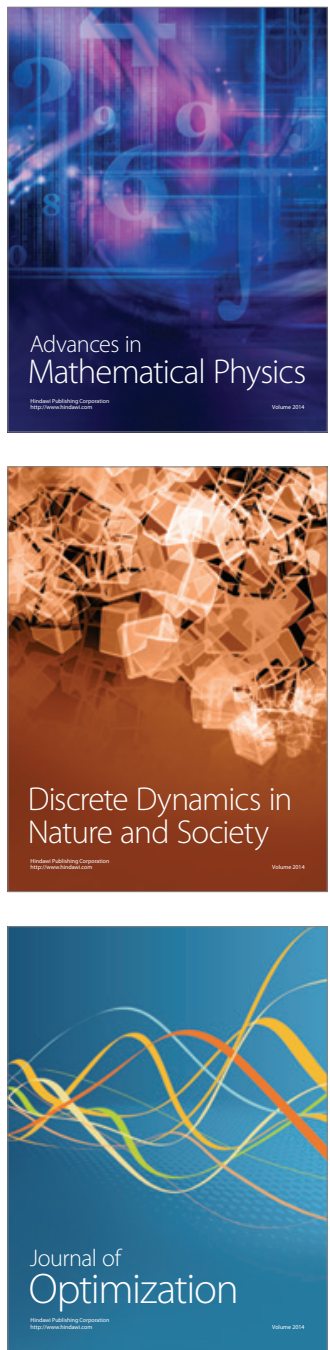